\title{
Fiery Fevers - An Epidemiological Evaluation and Management Strategies Experience from a Community Based Day Care Centre
}

\author{
Hemalalitha Shilpa Renduchintala*, Kodamarthy Vamsi Mohan, Sandhya Dixit \\ Sri K Kishan Rao Hospital, Vanasthalipuram, Hyderabad, India \\ Email address: \\ drshilparhl@gmail.com (H. S. Renduchintala),drvamsi311086@gmail.com (K. V. Mohan), sandhyadixit9@yahoo.com (S. Dixit) \\ ${ }^{*}$ Corresponding author
}

To cite this article:

Hemalalitha Shilpa Renduchintala, Kodamarthy Vamsi Mohan, Sandhya Dixit. Fiery Fevers - An Epidemiological Evaluation and Management Strategies Experience from a Community Based Day Care Centre. International Journal of Infectious Diseases and Therapy. Vol. 5, No. 1, 2020, pp. 17-22. doi: 10.11648/j.jiidt.20200501.14

Received: December 25, 2019; Accepted: March 5, 2020; Published: March 24, 2020

\begin{abstract}
Telangana has a long association with fiery fevers caused by mosquito bites. It is in fact in Telangana that Sir Ronald Ross discovered the malarial parasite in mosquito's gut in the month of August 1897 for which he was later awarded Nobel Prize in 1902. Malaria has been high on the differential diagnosis in patients presenting with fever in this region. However, over the last two decades there has been an increased incidence of mosquito borne viral diseases with India becoming endemic for Dengue and Chikungunya. The prevalence of these has been on a rise and Telangana region has been no exception. An unpredictable and sudden outbreak of fever cases prevailed from August to October 2019 in Hyderabad, most of them were viral fevers and among them Dengue emerged as a major toll, to a lesser extent Chikungunya and others. Objective of this study is to present a clear and comprehensive picture of the prevailing causes of such a fever outbreak in this specified time frame in our Diagnostics and Day care centre. Serum samples were collected from all fever cases and sent to laboratory and analysed according to the following criteria -age, gender, presenting complaints, lab evaluation etc. The results have been interpretated in the form of tables, figures and graphs reflecting the predominant cause of fevers. The study showed that majority of the cases were in the age group 20-30 years with a male predilection and significant number of patients showed leucopenia and thrombocytopenia even in non dengue and non chikungungya patients. This sudden emergence is being attributed to sparkling vector transmission due to an incessant rainfall during this time inhabiting breeders in stagnant waters. It also reemphasizes the need for regular public health maintenance programmes including removing of stagnant water, mosquito control, regular public awareness camps. Need of the hour would be for both governmental and nongovernmental agencies to work in coordination to reduce the disease burden.
\end{abstract}

Keywords: Vectorborne Diseases, Dengue, Chikungunya, Leucopenia and Arthralgia

\section{Introduction}

India has become endemic to vector borne viral diseases including Dengue and Chikungunya. These viral illnesses can cause significant morbidity and may also cause mortality if not promptly diagnosed and monitored for adverse complications. There has been a considerable increase in the number of patients presenting with high grade fever and intense arthralgia's and myalgia's during early monsoon period of 2019 in the Telangana region. This prompted us to take up this observational study to further understand the epidemiology of these diseases.

Objective of the study: Epidemiological evaluation of patients presenting with fever to a community based day care centre in the region of Telangana and observation of the disease pattern and response to management.

\section{Methods}

This is an observational prospective cohort study conducted at Sri K KishanRao Hospital Diagnostics and Day care centre Vanasthalipuram, Hyderabad, Telangana, India. 
Consecutive patients with fever as the presenting complaint for a three month period starting 01-08-2019 were enrolled in to the study.

Each patient was thoroughly evaluated by the attending physician and following investigations including full blood count, urea and electrolytes, thick and thin peripheral smears for malarial parasite, salmonella serology, urine analysis, ESR, blood for culture sensitivity and viral serology and a rapid test for Dengue were undertaken for all patients. Additional investigations including chest $-\mathrm{X}$ ray, ultrasound abdomen, sputum cultures etc. were undertaken where clinically appropriate.

Patient's symptomatology, vitals and examination findings were appropriately documented, initial supportive therapy was provided and patients with abnormal vitals, high risk features including hypotension, shock, tachycardia, and altered consciousness were promptly shifted to a hospital for inpatient management. Patients with leucopenia or severe thrombocytopenia who were otherwise stable were also advised hospital admission for close monitoring and ready

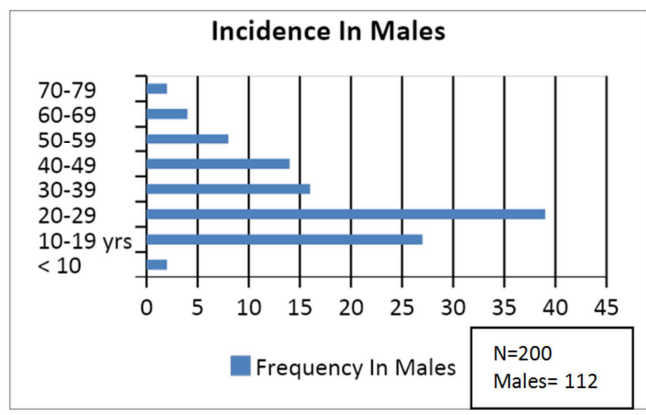

intervention where needed. In patients who tested positive for Dengue WHO criteria was strictly adhered to whilst advising hospital admission.

Patients were monitored and followed up regularly as guided by initial results, response to treatment and clinical progress.

\section{Results}

\subsection{Incidence According to Gender}

A total of 200 patients ( $66 \%$ male) were seen during the 3 month study period between 01-08-2019 to 31-10-2019 at Sri K KishanRao Hospital Diagnostics and Day care centre Vanasthalipuram, Hyderabad, Telangana, India.

As per the age groups in males, between 10 to 29 years, $58.9 \%$ cases were seen.

In females in the age group of 10 to 29 years, $51.1 \%$ cases are seen.

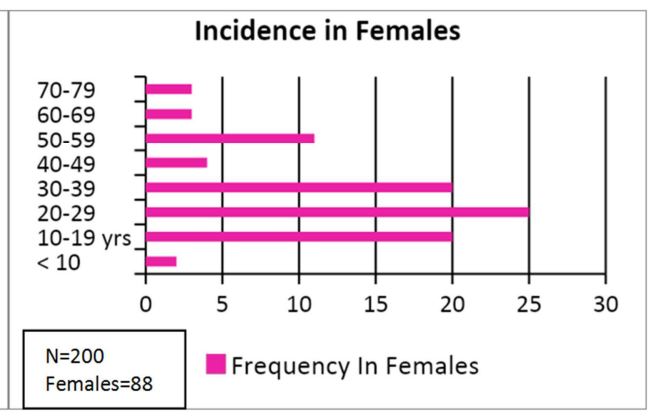

Figure 1. Incidence in males and females.

\subsection{Causes}

Etiologies included Dengue (19\% of cases of which males constituted around $60 \%)$, urinary tract infections $(10.5 \%$ female patients constituting around $80 \%$ ), Chikungunya (4\% majority of whom were female patients barring one case), Combined (dengue and chikungunya in $2 \%$ ), other causes included typhoid in one patient, upper respiratory tract infections, viral flu, gastroenteritis and fevers of non-specific etiology. None of the patients were positive for malarial parasite.

Dengue fever is commoner in males in contrast to females who have more predilection to chikungunya and combined.

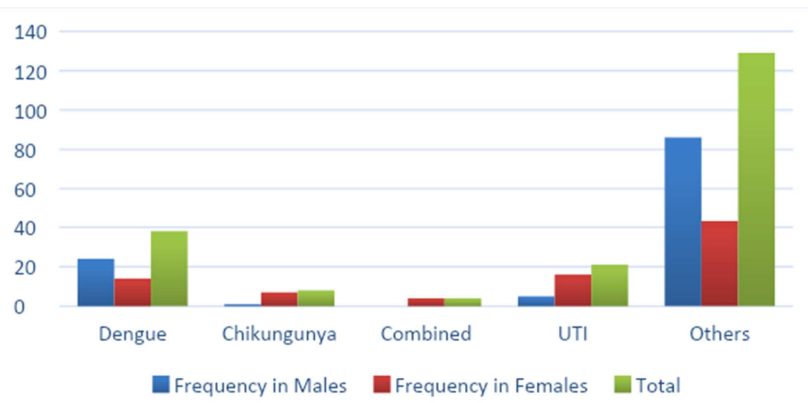

Figure 2. Underlying etiologies.

\subsection{Clinical Symptomology}

Fever was the presenting symptom in all these patients other symptomatology included aches and pains in 55\% and headache in $78 \%$, and viral exanthema in $16 \%$. Patients had their symptoms for an average of 2-3 days prior presentation and over fifty percent of patients gave history of selfmedication.

Though fever is the commonest presentation, aches and pains were the dominant symptoms in the study including headache which is $78 \%$.

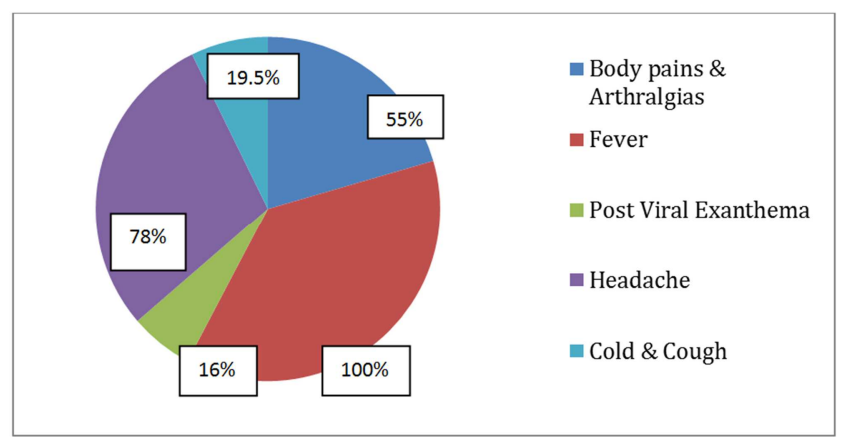

Figure 3. Percentage of presenting complaints. 


\subsection{Significant Lab Investigations}

Leucopenia was noted in $15 \%$ of cases of which male patients constituted $66 \%$ and thrombocytopenia was seen in $4.5 \%$ of patients of whom majority were males and severe thrombocytopenia with platelet counts $<5000$ was seen in two patients (one male patient).

Leucopenia is more dominant lab investigation report compared to thrombocytopenia

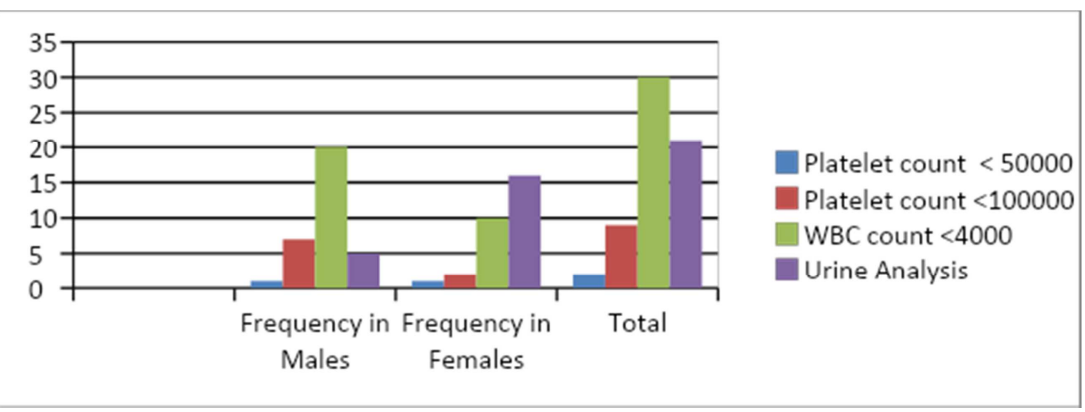

Figure 4. Salient lab reports analysis.

\subsection{Management}

$47 \%$ of the patients [94 out of 200] were managed on an out-patient basis. Complete symptom control was achieved in $55 \%$ of these patients at one week follow-up whilst $45 \%$ of patients continued to be symptomatic with myalgia and arthralgia which were treated with analgesics. $28 \%$ of patients [56 out of 200] were treated at a local hospital without any mortality and rest of the patients missed the follow up after initial outpatient visit.

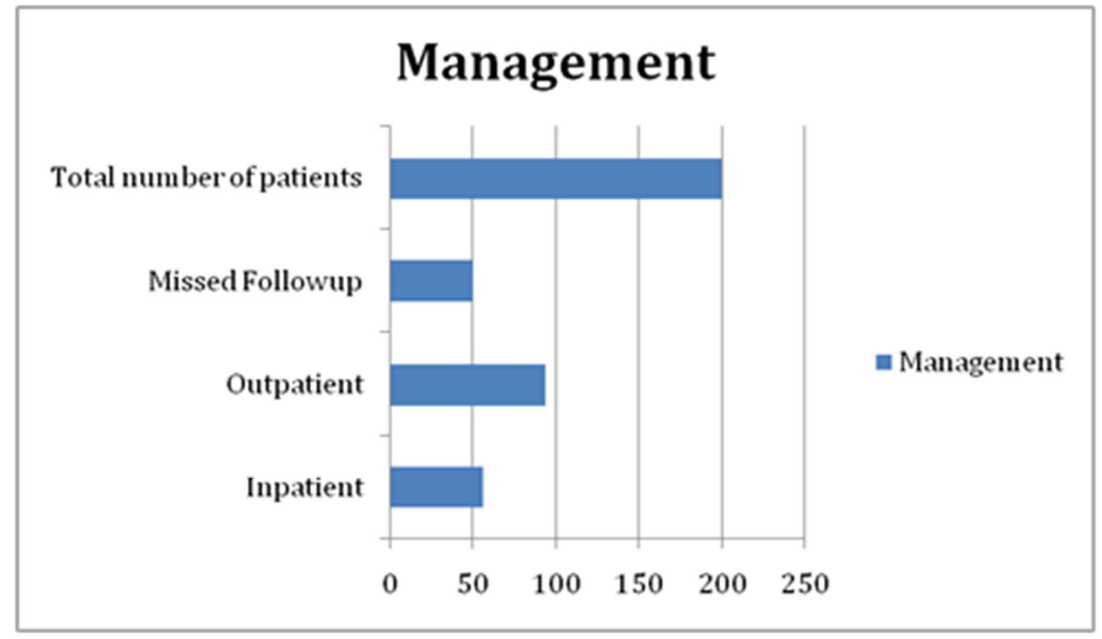

Figure 5. Modality of treatment setting and followup.

\subsection{Outcome}

There was no mortality amongst study patients. Average length of stay for admitted patients was less than 3 days. Complete recovery is seen in $45.5 \%$ of patients.

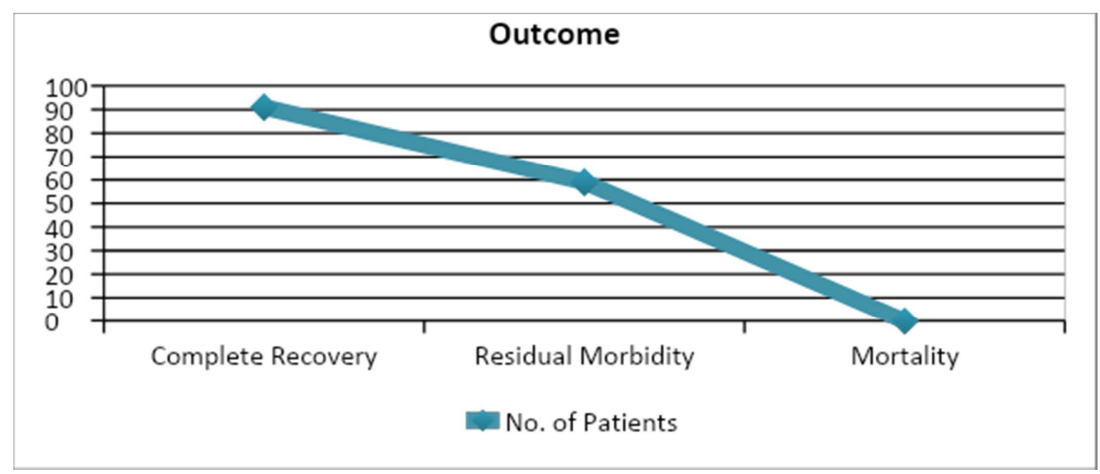

Figure 6. Patient outcomes. 


\section{Discussion}

Both Dengue and Chikungunya virus are RNA viruses that are vector borne and are spread by mosquito bites [1]. India has been endemic for these illnesses over last few decades [2].

Dengue can be caused by any of the four dengue virus serotypes: DENVs $1-4$ and is primarily transmitted to humans by the female Aedesmosquito [3]. Dengue can manifest as a spectrum ranging from asymptomatic to severely symptomatic cases [4-10] The symptoms can last for up to a week and patients need to be monitored for adverse prognostic features as this disease can result in significant morbidity and mortality if unmonitored. The World Health Organization classifies dengue into 2 major categories: dengue (with / without warning signs) and severe dengue. This has been a refinement from its previous classification mainly to help health practitioners to triage these patients for further hospitalization [11-13]. Mortality in patients with severe dengue is mainly due to plasma leaking, fluid accumulation, respiratory distress, severe bleeding, or organ impairment. Treatment is mainly supportive with analgesia, platelet transfusion if required and other supportive measures based on severity and end organ involved. Almost all states of India have become endemic to this disease, Telangana being no exception. Studies prior 2011 showed incidence of DENV serotypes 3 and 4 in this region [14] however more recent studies have reported incidence of all four strains [15].

Dengue has been shown to affect mainly young people in India. Dengue positivity was higher between the months of August and November, corresponding to monsoon and postmonsoon season in most states in India [16]. Mortality for the disease can be reduced to less than $1 \%$ when patients are monitored and appropriately treated. As per the National Vector borne Disease Control Program data, a total of 683,545 dengue cases and 2,576 deaths were reported in India during 2009-2017 giving a mortality rate of 0.38 [18]. However very low rates of mortality in this data have been hypothesized to be partly due to under reporting $[19,20]$.

This disease also poses considerable economic burden in India as studies seem to suggest huge financial implications with costs mounting up to USD 548 million according to one estimate [20-23].

Whilst various serological investigations including enzyme-linked immunosorbent assays (ELISA) and various reverse transcriptase-polymerase chain reaction (RT-PCR) methods are available for detection of DENV, commerciallyproduced rapid diagnostic tests which test for NS-1 (a virus produced protein) has been popular as the result is available in about 20 minutes [24, 25].

There has been periodic surge in these diseases during monsoon and post monsoon seasons due to excessive mosquito breeding during these times. There has been phenomenal increase in the incidence over the recent past partly contributed by the extended monsoons in 2019, with significant increase in reported cases of dengue and Chikungunya. Both of these are known to cause extensive symptomatology mainly arthralgia's and myalgia's that cause considerable morbidity. Due to residual symptoms in significant amount of the diseased patients as demonstrated in our cohort, this can lead to considerable work loss days and have significant financial implications particularly in under privileged sections of Indian society.

The vector borne diseases like dengue and chikungunya come in scores where mosquitoes are breeding. Wetlands, wet areas, temporary stagnant pools of water are the prerequisite factors for an increase in case load. One of the proposed factors for increase in the number of people affected is the urbanization of rural and semi urban areas with little planning for waste disposal.

Most of the efforts to control Dengue has been focussed on controlling the vectors by educating public at largeabout the need for prevention of mosquito breeding by environmental management and modification including disposing of solid waste properly covering, emptying and cleaning of domestic water storage containers regularly, using of personal household protection measures, such as window screens, repellents, insecticide treated materials, coils and vaporizers and various government programs including identification of high risk areas and use of insecticides as space spraying during outbreaks.

Whilst there is now a live attenuated Dengue vaccine which has been shown to be efficacious it comes with a caveat that it has been shown to be effective in seropositive individuals (persons previously infected by Dengue). In seronegative individuals it was noted that there is an increased risk of severe Dengue should patient develop first natural Dengue infection following vaccination [26, 27]. WHO now recommends vaccine for seropositive individuals [28-30]. Seropositivity in India in one study has been estimated over 50 percent. However use of Dengue vaccine has not yet been adapted as a routine practice in India.

Most of the reported studies in India about Dengue are mainly Hospital based studies. There is very sparse data about community based epidemiological studies [16]. Our study is unique that it is from a day care centre and comes closest to representing a community study. Our study shows incidence of Dengue around $20 \%$ in patients presenting with fever this is slightly lower than $38 \%$ (in patients with suspected Dengue) represented in some of the previous hospital based studies. This may be due to selection bias resulting from more severe and symptomatic cases presenting to tertiary care centres. Our study also shows that the incidence has been higher in young male population a finding previously reported in the studies from West Bengal [32, 33]. On the contrary, higher incidence of chikungunya was seen in females which is also found to be comparable to another study from Chennai and Vellore [34, 35].

The predilection of younger age groups both in men and women and the associated leucopenia and thrombocytopenia 
in severe cases is in keeping supressed immune states associated with these conditions.

In our series we also found a small proportion of patient that are infected both by DENV and Chikungunya virus this has also been reported in some of the previous studies [36].

Interestingly there has been no mortality in our cohort and this is in keeping with some of the previous studies which showed extreme reduction in mortality when patients are appropriately monitored for adverse prognostic features. We adhered strictly to WHO criteria for management of Dengue positive cases and admitted patients appropriately for further in-patient care. Our case series confirms the concept of community based management, observation and follow-up of Dengue positive cases with excellent outcomes when guidelines are strictly adhered and appropriate protocols are followed.

Our series also highlights the changing epidemiology of fiery fevers in urban India with no cases of malaria being found in this cohort. One of the other observations from our study was late presentation and self-medication amongst this cohort. Our study also highlights that there is a need to educate public at large about these diseases and highlight the need for early presentation which can in turn lead to timely diagnosis and identification of high risk cases.

Study limitations:

We acknowledge the small sample size of our study and we could not serotype Dengue positive cases due to constraints being a day care centre.

\section{Conclusion}

Basically diseases are multifactorial. Inspite of a particular etiology or an organism leading to a specific disease, environmental factors, herd immunity, presenting individuals age, gender and comorbidities influence the degree of morbidity and response to management. Though the epidemiology, lab investigations and management options are almost similar for every patient, the outcomes in each individual is varied.

Duration of the disease burden, loss of manhours leading to economic loss and associated psychosocial stress is an added concern.

With this background, we present this unique community based data which suggests changing epidemiology of fiery fevers with Dengue fever leading the list of fevers with different causative organisms. Our data also confirms the concept of community based diagnosis and management of fevers with WHO triage and management guidelines leading to excellent outcomes.

\section{Acknowledgements}

We sincerely acknowledge the efforts of our team of consultants, lab personnel and other hospital team members. And my heartfelt compassion to all our patients who came to our hospital with confidence in our medical team.

\section{References}

[1] Waggoner, J. J., et al. (2012), Viremia and Clinical Presentation in Nicaraguan Patients Infected With Zika Virus, Chikungunya Virus, and Dengue Virus. Clinical Infectious Diseases: 63 (12), 1584-1590.

[2] Chakravarti A., Arora R., Luxemburger C (2012). Fifty years of dengue in India. Trans R Soc Trop Med Hyg: 106, 273-282.

[3] Bhatt, S., et al.(2013). The global distribution and burden of dengue. Nature: 496 (7446), 504-507.

[4] Shu PY, Huang JH (2004). Current advance in dengue diagnosis. Clin Diagn Lab Immunol: 11, 642-50.

[5] Agarwal J, Kapoor G, Srivastava S, Singh KP, Kumar R, Jain A (2010). Unusual clinical profile of Dengue Infection in patients attending a tertiary care teaching hospital in north India. Int J Infect Dis: 14, 174-5.

[6] Chaturvedi UC, Kapoor AK, Mathur A, Chandra D, Khan AM, Mehrotra RML (1970). A clinical and epidemiological study of an epidemic of febrile illness with haemorrhagic manifestations which occurred at Kanpur, India, in 1968. Bull World Health Organ: 43 (2), 281.

[7] Chhina DK, Goyal O, Goyal P, Kumar R, Puri S, Chhina RS (2009). Haemorrhagic manifestations of dengue fever \& their management in a tertiary care hospital in north India. Indian $\mathrm{J}$ Med Res: 129 (6), 718-721.

[8] Duong, V., et al. (2015), Asymptomatic humans transmit dengue virus to mosquitoes. Proceedings of the National Academy of Sciences of the USA: 112 (47), 14688-14693.

[9] G. N. Malavige, S. Fernando, D. J. Fernando, and S. L. Seneviratne (2004). "Dengue viral infections," Postgraduate Medical Journal: 80 (948), 588-601.

[10] Pandey N, Nagar R, Gupta S (2012). Trend of dengue virus infection at Lucknow, north India: a hospital based study. Indian J Med Res: 136 (5), 862.

[11] World Health Organization. Dengue hemorrhagic fever: diagnosis, treatment and control. 1997.

[12] World Health, O., Dengue: Guidelines for Diagnosis, Treatment, Prevention and Control. Dengue: Guidelines for Diagnosis, Treatment, Prevention and Control. 2009, Geneva: World Health Organization. 1-147.

[13] Barniol J, Gaczkowski R, Barbato EV, da Cunha RV, Salgado D, Marti'nez E, et al (2011). Usefulness and applicability of the revised dengue case classification by disease: multi-centre study in 18 countries. BMC Infect Dis: 11, 106.

[14] Dash PK, Sharma S, Srivastava A, Santhosh SR, Parida MM, Neeraja M, et al (2011). Emergence of dengue virus type 4 (genotype I) in India. Epidemiol Infect: 139 (06), 857-61.

[15] Neeraja M, Iakshmi V, Teja VD, Lavanya V, Priyanka EN, Subhada K, et al (2014). Unusual and rare manifestations of dengue during a dengue outbreak in a tertiary care hospital in South India. Arch Virol: 159 (7), 1567-73.

[16] Ganeshkumar P, Murhekar MV, Poornima V, Saravanakumar V, Sukumaran K, Anandaselvasankar A, et al. (2018). Dengue infection in India: A systematic review and metaanalysis. PLoS Negl Trop Dis: 12 (7): e0006618. 
[17] National Vectorborne Disease Control Program, Directorate General of Health Services. http://nvbdcp.gov.in/DENGU1.html.

[18] Das S, Sarfraz A, Jaiswal N, Das P (2017). Impediments of reporting dengue cases in India. J Infect Public Health: 10, 494-498.

[19] Kakkar M (2012). Dengue fever is massively under-reported in India, hampering our response. BMJ: 19 (354), e8574-e8574.

[20] Murtola T, Vasan S, Puwar T, Govil D, Field R, Gong H, Bhavsar-Vyas A, Suaya J, Howard M, Shepard D, Kohli V, Prajapati P, Singh A, Mavalankar D (2010). Preliminary estimate of immediate cost of chikungunya and dengue to Gujarat, India. Dengue Bull: 34, 32-38.

[21] Kumar D, Garg S. Economic burden of dengue fever on households in Hisar district of Haryana state, India (2014). Int J Adv Med Health Res: 1 (2), 99-103.

[22] Garg P, Nagpal J, Khairnar P, Seneviratne SL (2008). Economic burden of dengue infections in India. Trans R Soc Trop Med Hyg: 102 (6), 570-7.

[23] Shepard DS, Halasa YA, Tyagi BK, Adhish SV, Nandan D, Karthiga KS, Chellaswamy V, Gaba M, Arora NK, INCLEN Study Group (2014). Economic and disease burden of dengue illness in India. Am Soc Trop Med \& Hygiene: 91 (6), 1235-42.

[24] Duthade MM, Damle AS, Bhakre JB, Gaikwad AA, Iravane JA, Jadhav A, et al (2015). The Study of Detection of Dengue NS1 Antigen and IgM Antibody by ELISA in and around Aurangabad, India. Int J Curr Microbiol App Sci: 4 (10), 416422 .

[25] Gopal KA, Kalaivani V, Anandan H (2016). Prevalence of Dengue Fever and Comparative Analysis of IgM and IgG Antibodies in Dengue Fever in Thoothukudi-Southern Coastal City, Tamil Nadu. Annals of International Medical and Dental Research: 2 (6), 4-7.

[26] Hadinegoro SR, Arredondo-GarcôÂa JL, Capeding MR, Deseda C, Chotpitayasunondh T, Dietze R, et al (2015). Efficacy and Long-Term Safety of a Dengue Vaccine in Regions of Endemic Disease. N Engl J Med: 373, 1195-206.
[27] Villar L, Dayan GH, Arredondo-Garcia JL, Rivera DM, Cunha R, Deseda C, et al (2015). Efficacy of a tetravalent dengue vaccine in children in Latin America. N Engl J Med: 372, 113-23.

[28] WHO (2016). Dengue vaccine: WHO position. Wkly Epidemiol Rec: 91, 349-64.

[29] WHO (2017). Updated Questions and Answers related to the dengue vaccine Dengvaxiaanditsuse. http://www.who.int/immunization/diseases/dengue/q_and_a_d engue_vaccine_dengvaxia_use/en/.

[30] WHO (2018). Revised SAGE recommendation on use of dengue vaccine. http://www.who.int/immunization/diseases/dengue/revised_S AGE_recommendations_dengue_vaccines_apr2018/en/.

[31] Garg S, Chakravarti A, Singh R, Masthi NRR, Goyal RC, Jammy GR, et al (2017). Dengue serotype-specific seroprevalence among 5- to 10-year-old children in India: a community-based cross-sectional study. Int J Infect Dis: 54, $25-30$.

[32] Bandyopadhyay B, Bhattacharyya I, Adhikary S, Konar J, Dawar N, Sarkar J, et al (2012). A comprehensive study on the 2012 dengue fever outbreak in Kolkata, India. ISRN Virol: 2013,5 .

[33] E. Gupta, L. Dar, G. Kapoor, and S. Broor (2006), "The changing epidemiology of dengue in Delhi, India," Virology Journal: $3,92$.

[34] Balasubramaniam SM, Krishnakumar J, Stephen T, Gaur R, Appavoo NC (2011). Prevalence of Chikungunya in urban field practice area of a private medical college, Chennai. Indian J Community Med: 36, 124-7.

[35] Selvavinayagam TS (2007). Chikungunya fever outbreak in Vellore, south India. Indian J of Community Medicine: 32 (4), 286-7.

[36] Barde PV, Shukla MK, Bharti PK, Kori BK, Jatav JK, Singh $\mathrm{N}$ (2014). Co-circulation of dengue virus serotypes with chikungunya virus in Madhya Pradesh, central India. WHO South-East Asia Journal of Public Health: 3 (1), 36. 